\title{
Propagation of scrapie in peripheral nerves after footpad infection in normal and neurotoxin exposed hamsters
}

\author{
Christine KratzeL*, Jessica MaI, Kazimierz Madela, Michael BeEKes, \\ Dominique KRÜGER*
}

Robert Koch-Institut, P24 - Transmissible Spongiforme Enzephalopathien, Nordufer 20, 13353 Berlin, Germany

(Received 29 May 2006; accepted 1 September 2006)

\begin{abstract}
As is known from various animal models, the spread of agents causing transmissible spongiform encephalopathies (TSE) after peripheral infection affects peripheral nerves before reaching the central nervous system (CNS) and leading to a fatal end of the disease. The lack of therapeutic approaches for TSE is partially due to the limited amount of information available on the involvement of host biological compartments and processes in the propagation of the infectious agent. The in vivo model presented here can provide information on the spread of the scrapie agent via the peripheral nerves of hamsters under normal and altered axonal conditions. Syrian hamsters were unilaterally footpad (f.p.) infected with scrapie. The results of the spatiotemporal ultrasensitive immunoblot-detection of scrapie-associated prion protein $\left(\mathrm{PrP}^{\mathrm{Sc}}\right)$ in serial nerve segments of both distal sciatic nerves could be interpreted as a centripetal and subsequent centrifugal neural spread of $\mathrm{PrP}^{\mathrm{Sc}}$ for this route of infection. In order to determine whether this propagation is dependent on main components in the axonal cytoskeleton (e.g. neurofilaments, also relevant for the component ' $a$ ' of slow axonal transport mechanisms), hamsters were treated -in an additional experiment- with the neurotoxin $B, \beta$ '-iminodiproprionitrile (IDPN) around the beginning of the scrapie infection. A comparison of the Western blot signals of $\operatorname{PrP}^{\mathrm{Sc}}$ in the ipsilateral and in the subsequently affected contralateral sciatic nerve segments with the results revealed from IDPN-untreated animals at preclinical and clinical stages of the TSE disease, indicated similar amounts of PrPSc . Furthermore, the mean survival time was unchanged in both groups. This in vivo model, therefore, suggests that the propagation of $\mathrm{PrP}^{\mathrm{Sc}}$ along peripheral nerves is not dependent on an intact neurofilament component of the axonal cytoskeleton. Additionally, the model indicates that the spread of $\mathrm{PrP}^{\mathrm{Sc}}$ is not mediated by the slow component ' $a$ ' of the axonal transport mechanism.
\end{abstract}

scrapie / PrPSc / nerve / footpad / IDPN

\section{INTRODUCTION}

Transmissible spongiform encephalopathies (TSE), such as the CreutzfeldtJakob Disease (CJD) in humans, bovine spongiform encephalopathy (BSE) in cattle or scrapie in sheep are fatal diseases

* Corresponding authors:

kratzelc@rki.de, kruegerd@ rki.de of the central nervous system (CNS) in mammalians. The etiological classification differentiates between genetic, acquired or sporadic forms.

The key molecular pathological event, characteristic to all forms of TSE, seems to have been elucidated: The normal host cellular prion protein $\left(\mathrm{PrP}^{\mathrm{C}}\right)$ converts into a disease-specific form [11], that is resistant 
to proteinase $\mathrm{K}$ digestion [42]. The glycoprotein $\mathrm{PrP}^{\mathrm{C}}$ is strongly expressed by cells of the nervous and lymphoreticular systems. During biogenesis, $\operatorname{PrP}^{\mathrm{C}}$ migrates to the outer plasma membrane where it is anchored by glycosyl-phosphatidylinositol [47]. In vivo studies showed that $\operatorname{PrP}^{C}$ is transported in nerves by axonal anterograde and retrograde transport mechanisms $[8,36,45]$. However, the normal function of $\mathrm{PrP}^{\mathrm{C}}$ is still unclear.

The abnormal scrapie specific prion protein $\left(\mathrm{PrP}^{\mathrm{Sc}}\right)$ is a biochemical marker for TSE and is linked with infectivity. This fact coupled with the failure to successfully transmit the disease in $\operatorname{PrP}^{\mathrm{C}}$ knock-out mice models [11], the in vitro generation of infectious $\operatorname{PrP}[13,32]$ and the futile search for specific prion-associated nucleic acids are all strong indicators that $\mathrm{PrP}^{\mathrm{Sc}}$, according to the protein-only hypothesis [42], is the causative TSE agent. Post-mortem histological examinations of brain tissue of clinically affected individuals revealed astrocytosis and vacuolar degeneration besides the detection of antibody-positive granular deposits of $\mathrm{PrPSc}^{\mathrm{Sc}}$.

The natural route of infection for acquired forms of TSE is the peripheral uptake of the infectious agent. Known sources of iatrogenic CJD in humans are transmission via infected grafts (cornea or meninx), via neurosurgical devices or transmission by the administration of human pituitary hormones [10]. There is some evidence that TSE can be transmitted by blood transfusion from infected donors $[33,40]$. A large number of in vivo experiments suggest that the most important epidemiological modus for scrapie, BSE or variant-CJD (vCJD) transmission is the oral route $[2,39]$. The endemic appearance of Kuru in Papua New Guinea is due to endocannibalistic rites [1]. Nevertheless, in vivo experiments showed successful transmission of TSE-disease not only by oral, intracerebral, intravenous or intraperitoneal application of infectivity but also by subcutaneous injection, through defect mucosa $[5,12]$ or, even very effectively, by scarification of the intact skin [48].

The sequential detection of abnormal prion protein in lymphoreticular tissue and along neural projections to the CNS after peripheral infection in various animal models led to the conclusion that these tissues are involved in the spread of the TSE agent to the CNS $[3,7,17,28,35,44$, 46]. The sciatic nerve is a useful structure for monitoring the centripetal neural spread of the scrapie agent [29] and rapid disease onset was observed after intrasciatic injections of strains of transmissible mink encephalopathy (TME) in rodents [4]. Kimberlin [29] debated that the velocity of the spread of the scrapie agent to the CNS is comparable to the velocity of slow axonal transport. In all eukaryotic cells, including neurons, intracellular transport of membranous and nonmembranous cargoes (e.g. vesicles, proteins, mRNA) is of vital importance. Axonal transport proceeds in a fast or slow manner in anterograde or retrograde direction (from the cell body to the axon tip and vice versa). With respect to the rate and nature of moving structures, the slow axonal transport can be distinguished in two components. The component ' $a$ ' of slow axonal transport moves material bidirectionally at an overall rate of around $1 \mathrm{~mm}$ per day. Amongst moving material are the main proteins of the axonal cytoskeleton, neurofilament proteins and tubulin, whereby cytoskeletal polymers act as carrier structures [9]. It is still unclear whether the development of TSE interferes with the axonal transport of neurofilaments [20].

In order to answer the question whether axonal transport mechanisms are involved in the spread of the infectious agent along axons, previous studies were carried out in four-repeat tau over-expressing mice and in dynein mutant mice models [25, 31]. The results suggested that the fast host retrograde axonal transport mechanism did 
not serve as a "ferry" for the centripetal propagation of $\mathrm{PrP}^{\mathrm{Sc}}$.

In this study Syrian hamsters were unilaterally footpad (f.p.) infected with $263 \mathrm{~K}$ scrapie, approximating a natural route of infection, in order to characterize the normal neural pathway of the disease for this route. In a further experiment, animals were treated with iminodiproprionitrile (IDPN), known to disorganize neurofilaments and axonal transport mechanisms $[14-16,19,21,23,30,37,38]$. We analyzed Western blot signals of protease resistant PrP from the sciatic nerve segments of f.p. infected animals in non- and IDPN-exposed groups at the preclinical and clinical stages of scrapie. Furthermore, we compared the time required to reach the terminal stage of disease, to clarify whether the assumed centripetal spread of the infectious agent to the CNS and its subsequent centrifugal travel to the periphery are dependent on the intact neurofilament component of the axonal cytoskeleton.

\section{MATERIALS AND METHODS}

\subsection{Animal inoculations}

The animal experiments complied with German legal provisions.

Adult male and female Syrian hamsters were sedated with isofluran and f.p. infected with $20 \mu \mathrm{L}$ of a $2 \%$ (wt/vol) terminally ill 263K-hamster brain homogenate or mock-infected with normal hamster brain homogenate in the sole of the right foot by injection using a 27 gauge cannula. The animals were regularly monitored for clinical signs of scrapie ranging from an increased sensitivity to touch and sound to muscle tremor, kiphosis, ataxia and rapid weight loss.

\subsection{Tissue collection for spatiotemporal $\mathrm{PrP}^{\mathrm{Sc}}$ analysis in sciatic nerves after f.p. infection}

The hamsters were sacrificed by $\mathrm{CO}_{2}$ asphyxiation at different time points following infection or when terminally ill. Samples of both distal sciatic nerves were divided into quarters weighing $3.6 \pm 1.4 \mathrm{mg}$ (mean $\pm \mathrm{SD}$ ) for Western blot analyses. Specimens were TBSwashed and frozen at $-80{ }^{\circ} \mathrm{C}$ immediately after preparation until further processing. To avoid cross-contamination, the instruments used for the preparation of samples were carefully cleaned after removal and processing of each specimen.

Western blot results were reported for samples at the following time points: 2 days post infection (dpi, $n=2$ ), 42 dpi $(n=5), 60$ dpi $(n=2), 70$ dpi $(n=6)$, 80 dpi $(n=6), 90$ dpi $(n=7), 100$ dpi $(n=4)$ and when terminally ill with severe symptoms at $100 \mathrm{dpi}(n=6)$ and 119 dpi $(n=2)$, and for samples of mockinfected hamsters manifesting no clinical signs which were sacrificed at 42 dpi and at $>100$ dpi.

Other animals were transcardially perfused with periodate-lysine-paraformaldehyde (PLP [34]); nerve and brain samples were removed and post-fixed for $4 \mathrm{~h}$ in PLP or the removed tissue was directly immersion fixed for a maximum of $48 \mathrm{~h}$ with neutral buffered $3.7 \%$ formalin. Tissue samples for immunohistochemistry (IHC) were taken $42 \mathrm{dpi}, 70 \mathrm{dpi}$, $80 \mathrm{dpi}, 90 \mathrm{dpi}, 100 \mathrm{dpi}$ (a total of $n=$ 4 preparations for every time point) and when terminally ill at 104 dpi and 110 dpi ( $n=2$, respectively). After incubation in phosphate-buffered saline, tissue samples were transferred to $70 \%$ ethanol, processed in a tissue processor and subsequently embedded in paraffin wax.

\subsection{Chronic IDPN intoxication and spatiotemporal tissue collection for PrPSc $^{\text {analysis }}$}

A tolerable treatment regime for IDPN intoxication was determined in preliminary tests. Hamsters were f.p. infected and treated intraperitoneally (i.p.) five times 
with IDPN (20 mg/100 g). The first injection was administrated 10 days before and the final injection 28 days after infection. Before administration, IDPN was diluted in saline and the $\mathrm{pH}$ was adjusted with hydrochloric acid to 7.4. Nerve tissue of a mock-infected, a scrapie-infected and a scrapie-infected IDPN-intoxicated hamster at 42 dpi (PLP fixed as described above) were the controls for immunohistological analysis to confirm the effect of the intoxication.

At 45 dpi peripheral blood of 6 untreated and 7 IDPN-treated animals was collected by puncturing the retrobulbar plexus. White blood cells were quantitatively and qualitatively compared by counting using a hemocytometer and by Giemsa staining of a blood smear.

Analogous to untreated hamsters, the IDPN-treated animals were sacrificed at different time points following infection or when terminally ill. Samples of both distal sciatic nerves were divided into quarters weighing $3.4 \pm 1.3 \mathrm{mg}$ (mean $\pm \mathrm{SD}$ ) for Western blot analyses. Specimens were TBS-washed and frozen at $-80{ }^{\circ} \mathrm{C}$ immediately after preparation until further processing. Western blot results were reported for samples at the following time points: 42 dpi $(n=4), 60$ dpi $(n=4), 70$ dpi $(n=$ 4), 80 dpi $(n=4), 90$ dpi $(n=4), 100$ dpi $(n=2)$ and when terminally ill with severe symptoms at 109 and $126 \mathrm{dpi}(n=$ 2). Mock-infected and IDPN-treated hamsters without clinical signs were sacrificed 42 dpi and at $>100$ dpi. For histological investigations, nerve and brain samples were removed 42 dpi $(n=4), 60$ dpi $(n=4)$, 70 dpi $(n=4), 80$ dpi $(n=4), 90$ dpi, 100 dpi $(n=3)$ and when terminally ill at 112 dpi $(n=1)$, fixed in PLP or formalin and processed as described above.

The incubation times until the development of terminal scrapie disease were determined in separate groups with IDPN-treated $(n=7)$ or nontreated animals $(n=4)$.

\subsection{Western blot}

For positive controls, nerve samples of uninfected animals were spiked with $10 \mu \mathrm{L}$ of $0.1 \%$ 263K-hamster brain homogenate (corresponding to $10^{-5} \mathrm{~g}$ of scrapie brain homogenate) prepared by ultrasonification of $1 \mathrm{~g}$ brain tissue of a terminally ill donor hamster in $10 \mathrm{~mL}$ Tris-buffer, $\mathrm{pH}$ 7.4. All nerve samples were incubated at $37{ }^{\circ} \mathrm{C}$ for $60 \mathrm{~min}$ with $500 \mu \mathrm{L} 0.25 \%$ collagenase in TBS buffer containing $2 \mathrm{mM}$ $\mathrm{CaCl}_{2}$. After halting the reaction by adding $10 \mu \mathrm{L} 250 \mathrm{mM}$ EDTA, tissue-extraction of $\mathrm{PrP}^{\mathrm{Sc}}$ in the form of the protease resistant 27-30 kD fragment (PrP27-30) was initiated in line with a previously published protocol [6], including the use of proteinase $\mathrm{K}(\mathrm{PK})$ to destroy normal $\mathrm{PrP}^{\mathrm{C}}$. The final pellets were resuspended with $20 \mu \mathrm{L}$ of $2 \times$ sample loading buffer and heated to $100{ }^{\circ} \mathrm{C}$ for $10 \mathrm{~min}$ prior to Western blotting using the monoclonal antiPrP antibody (mAb) 3F4 [27] and an alkaline phosphatase-conjugated secondary goat-anti-mouse antibody. Sodium dodecyl sulfate-polyacrylamide gel electrophoresis (SDS-PAGE) and Western blot analyses were performed as described elsewhere $[6,49]$. Antibody binding was visualized using CDP-Star (Tropix) as a chemoluminescent substrate for alkaline phosphatase and a Kodak X-OMAT AR film, exposed for 30-60 min.

\subsection{Immunohistochemistry}

For the detection of granular $\mathrm{PrP}^{\mathrm{Sc}}$, $2 \mu \mathrm{m}$ thick paraffin slides were pretreated with guanidiniumthiocyanate after deparaffinization and rehydration and heating in citrate buffer in a microwave. After blocking with $0.2 \%$ (wt/vol) casein (Tropix), the slides were first incubated with mAb 3F4, diluted 1:250 in blocking solution for $90 \mathrm{~min}$ at room temperature followed by incubation for $60 \mathrm{~min}$ at room temperature with an alkaline phosphatase-conjugated 
goat-anti-mouse antibody (DakoCytomation), diluted $1: 500$ in blocking solution. Immunostaining was performed using new fuchsine as substrate for alkaline phosphatase. On selected sections, 3F4 binding was visualized using a biotinconjugated goat-anti-mouse antibody, the avidin biotin-complex (Vectastain) technique and with diaminobenzidine (DAB) as a chromogen. DAB-stained sections were pre-treated with amino acid after deparaffinization. Subsequently, the slides were counterstained with hemalaun.

To verify the effects of IDPN, sections of sciatic nerve tissues were stained using, as the first antibody, a marker for neurofilaments (mAb 2F11, DakoCytomation), diluted 1:100 in phosphate-buffered saline and a fluorescent (Oregon green) labelled secondary antibody, diluted 1:100. 2F11 reacts with the phosphorylated form of a $70 \mathrm{kD}$ component of the three neurofilament major polypeptide units. An uninfected/untreated animal and an uninfected/ IDPN-treated animal as well as a $263 \mathrm{~K}$ infected/IDPN-treated animal were compared at $42 \mathrm{dpi}$.

\section{RESULTS}

\subsection{Spatiotemporal $\mathrm{PrP}^{\mathrm{Sc}}$ analysis in sciatic nerves after f.p. infection}

After unilateral infection in the right foot pad of hamsters with $263 \mathrm{~K}$ brain homogenate, the scrapie specific clinical phase started with the symptom of muscle tremor around $78 \mathrm{dpi}$.

To determine the distribution of $\mathrm{PrP}^{\mathrm{Sc}}$ in nerve tissue at different time points, quarters of both distal sciatic nerves (femural level) were collected and Western blots of the nerve segments were performed after extraction and PK digestion in order to detect only protease resistant $\mathrm{PrP}^{\mathrm{Sc}}$ in the form of PrP27-30. Figure 1 shows the typical results for the Western blot detection of PrP27-30 at different time points in serial segments of ipsilateral (lanes 2-5 in
A-G) and contralateral sciatic nerves (lanes 2-5 in H-N) of one animal, respectively. Nerve segments and spiked nerve segments of uninfected animals served as negative and positive controls for the PrP27-30 extraction procedure. Additionally, controls for blotting efficiency (lane 9 in A-N) were carried out to align the signal intensities. No noticeable signals for PrP27-30 in segments of both nerves were detectable 2 dpi (data not shown) and 42 dpi (Fig. 1). The f.p. infection led to the first clear detection of PrP27-30 in the ipsilateral sciatic nerves at $60 \mathrm{dpi}$, earlier than in contralateral nerves (around $80 \mathrm{dpi}$ ). The time frame from finding the first weak signals up to finding strong signals in the nerves was very short (about 10-20 days). Western blot results indicate an increasing level of $\mathrm{PrP}^{\mathrm{Sc}}$ in the sciatic nerves at the respective site with time, whereby signal intensities of segments of one nerve did not differ substantially.

Immunohistological examinations of the brain revealed the tendency for the region of the contralateral red nucleus $(\mathrm{RN})$, correlating with the ipsilateral sciatic nerve, to show stronger granular PrPimmunoreactive signals than the red nucleus of the other side (Fig. 2).

Granular PrP-positive deposits could be detected very rarely in the sciatic nerve. Figure 3 shows a cross section through the sciatic nerve with central axons, peripherally surrounded by myelin sheats. The light microscopical investigations indicated that the deposits were located at the axonal periphery, in a close position to the myelin sheath.

\subsection{Effects of IDPN-treatment, spatiotemporalPrPSc analysis in sciatic nerves after f.p. infection in neurotoxin-exposed animals and survival after infection}

The IDPN-dose and treatment regime were well tolerated in all animals. One 


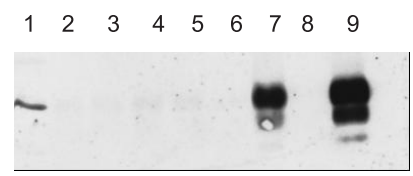

A. 42 dpi

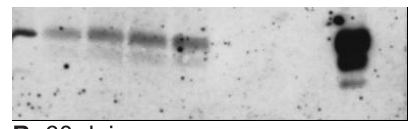

B. $60 \mathrm{dpi}$

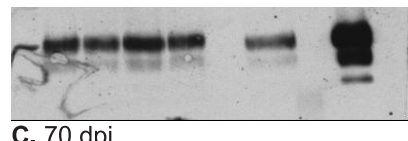

C. $70 \mathrm{dpi}$

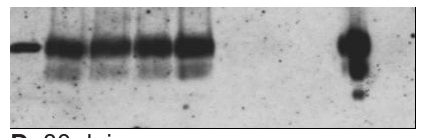

D. $80 \mathrm{dpi}$
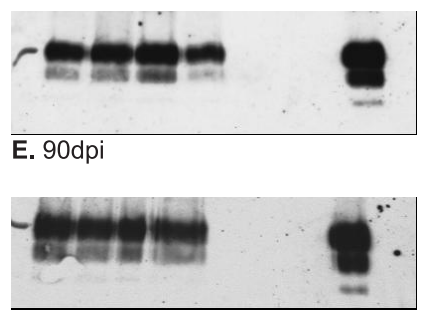

F. 100 dpi

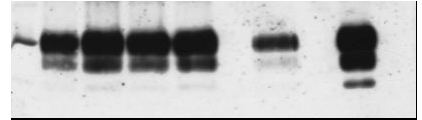

G. 100 dpi (terminally ill) $\begin{array}{lllllllll}1 & 2 & 3 & 4 & 5 & 6 & 7 & 8 & 9\end{array}$

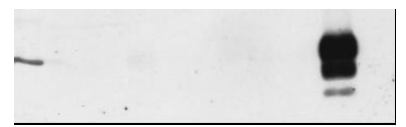

H. $42 \mathrm{dpi}$

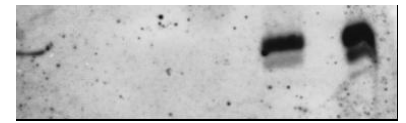

I. $60 \mathrm{dpi}$

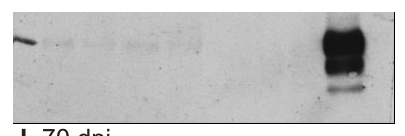

J. $70 \mathrm{dpi}$

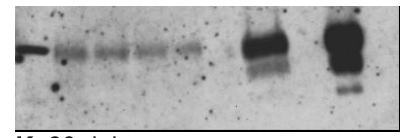

K. 80 dpi

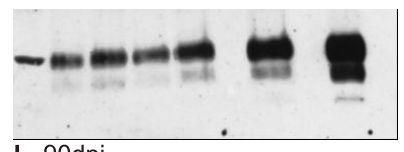

L. $90 \mathrm{dpi}$

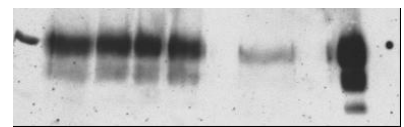

M. $100 \mathrm{dpi}$

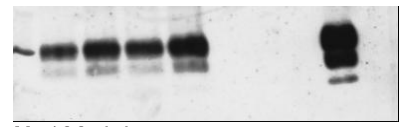

N. $100 \mathrm{dpi}$

Figure 1. Detection of PrP27-30. Representative Western blots of ipsilateral (A-G) and contralateral $(\mathrm{H}-\mathrm{N})$ sciatic nerve tissue of $263 \mathrm{~K}$ f.p. infected hamsters for indicated time points after infection. Lane 1: marker; lanes 2-5: sciatic nerve quarters from proximal to distal of the same individual at the indicated time point (samples correspond to the following amounts of tissue (mg): A2, 2.4; A3, 2.1; A4, 2.9; A5, 4.7; B2, 3.3; B3, 3.2; B4, 3.3; B5, 5.2; C2, 2.4; C3, 2.3; C4, 2.2; C5, 8.7; D2, 3.0; D3, 2.6; D4, 3.1; D5, 6.1; E2, 4.1; E3, 3.9; E4, 4.7; E5, 5.5; F2, 4.5; F3, 2.2; F4, 4.0; F5, 5.5; G2, 2.9; G3, 2.6; G4, 3.9; G5, 3.0; H2, 3.1; H3, 3.0; H4, 2.2; H5, 5.5; I2, 5.5; I3, 2.8; I4, 4.2; I5, 4.8; J2, 3.0; J3, 2.6; J4, 2.4; J5, 2.8; K2, 3.0; K3, 2.6; K4, 3.0; K5, 3.9; L2, 3.1; L3, 3.5; L4, 3.6; L5, 10.5; M2, 2.8; M3, 1.9; M4, 2.2; M5, 4.8; N2, 3.4; N3, 3.8; N4, 2.6; N5, 4.0); lane 7 (A, C, $\mathrm{G}, \mathrm{I}, \mathrm{K}, \mathrm{L}, \mathrm{M}$ ): spiked processing controls, $10^{-5} \mathrm{~g} 263 \mathrm{~K}$ brain homogenate (samples correspond to the following amounts of normal sciatic nerve tissue (mg): A7, 7.5; C7, 2.3; G7, 2.6; I7, 6.4; K7, 2.5; L7, 1.5; M7, 2.7); lane 7 (B, D, E, F, H, J, N): uninfected nerves as controls for PK digestion (samples correspond to the following amounts of normal sciatic nerve tissue (mg): B7, 2.6; D7, 2.8; $\mathrm{E} 7,1.8 ; \mathrm{F7}, 2.6 ; \mathrm{H7}, 2.9 ; \mathrm{J} 7,2.2 ; \mathrm{N} 7,2.5)$; lane 9: blot controls, $10^{-6} \mathrm{~g} 263 \mathrm{~K}$ brain homogenate. Lanes 6 and 8 were loaded with LPP. 


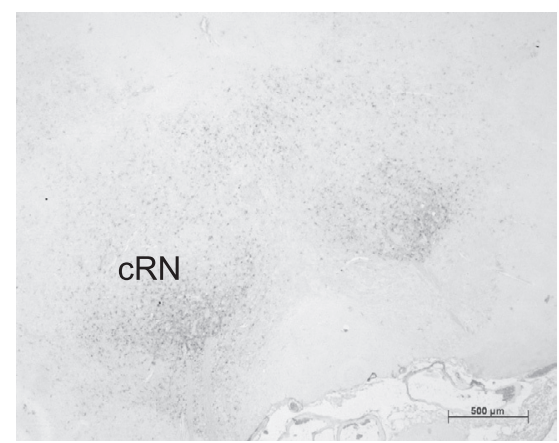

Figure 2. Representative micrograph for immunohistological staining of a $6 \mu \mathrm{m}$ brain section of a f.p. infected hamster 70 dpi using mAb 3F4 and DAB as chromogen, without counterstaining. Brown deposits for $\mathrm{PrP}^{\mathrm{Sc}}$ accumulate early in both red nuclei. There are slightly stronger deposits in the contralateral red nucleus (cRN). (A color version of this figure is available at www.edpsciences.org/vetres.)

observed side effect was the occurrence of temporary salivation 30 min after i.p. injection. One week after the last IDPNapplication (45 dpi), peripheral blood of separated animals was collected for monitoring general inflammatory side effects. The number and morphology of white blood cells were comparable in IDPNtreated and untreated animals (data not shown).

In further selected animals, tissue samples for immunohistological examinations were taken two weeks after the last IDPNapplication to verify the neural effects of the neurotoxin. Immunolabeling of neurofilaments using the mAb 2F11 showed that peripheral nerve tissue of IDPN-exposed hamsters had a stronger immunoreactivity than nerve tissue of untreated animals (Fig. 4).

After scrapie f.p. infection, the diseasespecific clinical phase started in the IDPNtreated animals, similar to the non-IDPNtreated animals, with symptoms of muscle tremor around $80 \mathrm{dpi}$.

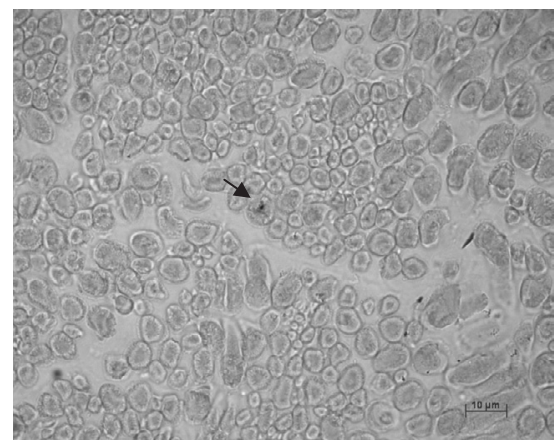

Figure 3. Representative micrograph for immunohistological staining of a $1 \mu \mathrm{m}$ ipsilateral sciatic nerve section of a f.p. infected animal (PLP-fixed tissue) at 80 dpi using mAb 3F4 and new fuchsine as chromogen. Red deposit (arrow) for $\mathrm{PrP}^{\mathrm{Sc}}$. (A color version of this figure is available at www.edpsciences.org/vetres.)

Figure 5 indicates no difference in survival after scrapie infection in non-treated and IDPN-treated animals (106 \pm 5 dpi and $105 \pm 4$ dpi, respectively, mean \pm SD).

Temporal Western blot signal intensities for PrP27-30 in serial nerve segments at all time points indicated comparable amounts of $\mathrm{PrP}^{\mathrm{Sc}}$ in analogous sciatic nerve segments in IDPN- and non-IDPN-treated animals for both ipsilateral or contralateral nerves (Fig. 6, compare with Fig. 1).

\section{DISCUSSION}

We chose an in vivo model mimicking injuries as a possible natural exposure to the TSE agent [48]. However, peripheral uptake via ingestion is the most likely route of exposure for several TSE diseases. Experimental oral infection of hamsters with $263 \mathrm{~K}$ scrapie required high doses and resulted in relatively long incubation periods until the terminal stage of the disease was reached $[43,49]$. In contrast, footpad infection with lower doses showed a relatively short mean incubation time with small variance, characterizing this route as 

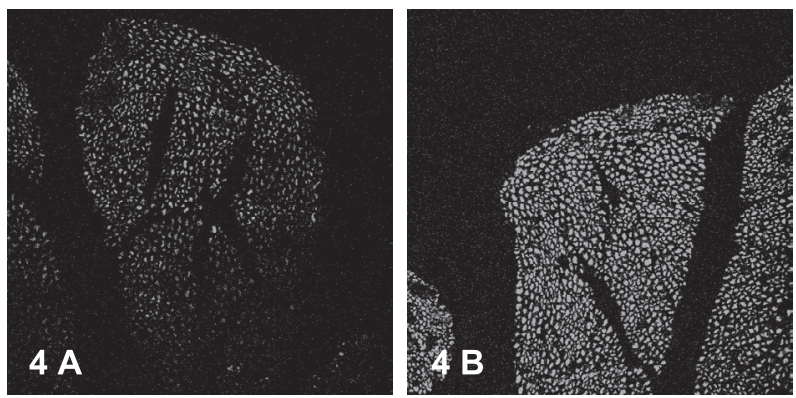

Figure 4. Cross sections of the distal sciatic nerve of an untreated animal (A) and an IDPN-treated animal (B). Immunostaining with a neurofilament antibody showed strong immunoreactivity in the IDPN-treated animal (confocal laser scanning microscopy, magnification $\times 400$.) (A color version of this figure is available at www.edpsciences.org/vetres.)

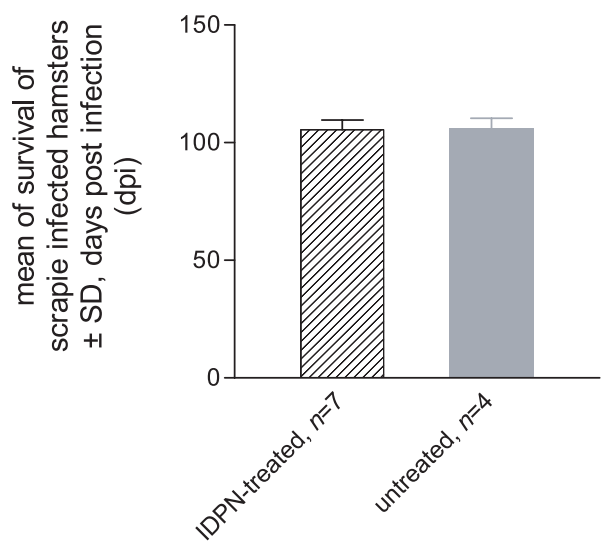

Figure 5. Mean survival time (dpi) after footpad scrapie infection with $20 \mu \mathrm{L} 2 \% 263 \mathrm{~K}$ brain homogenate in neurotoxin exposed and nonexposed hamsters

very effective. Therefore, skin lesions or injuries may be a relevant natural site of entry for the TSE agent.

Western blot analyses of protease resistant prion protein in both distal sciatic nerves and the immunohistochemical detection of $\mathrm{PrP}^{\mathrm{Sc}}$ in the corresponding brain areas after unilateral footpad infection with the scrapie agent (Figs. 1 and 2) pointed to the postulated centripetal propagation to the CNS and to the subsequent centrifugal spread of $\mathrm{PrP}^{\mathrm{Sc}}$ along nerval projections:
Cell bodies of the sciatic nerves are localized in the spinal cord. Corresponding areas in the brain of the sciatic nerve are the red nuclei. As most prominent projections, nerve fibers arising in the cells of RN cross ventral to the nuclei and course within the lateral funiculi of the spinal cord (Tractus rubrospinalis).

Spread along nerval projections was also suggested by Kimberlin [29] and Bartz [4] for the intrasciatic route of experimental scrapie- or TME-infection. Moreover, Kimberlin [29] assumed that the intrasciatic route bypasses extraneural agent replication. Nevertheless, for the footpad route, extraneural loci of agent replication cannot be ruled out since early and continuous accumulation of $\mathrm{PrP}^{\mathrm{Sc}}$ could also be detected in the regional lymphoid system (data not shown). However, up to now no method has been available to differentiate between the exogenously administered and endogenously formed pathological prion protein.

Our immunohistological investigations of the distal sciatic nerve concerning the localisation of $\mathrm{PrP}^{\mathrm{Sc}}$ rarely showed granular PrP-positive deposits, located apparently between the myelin sheath and axon. However, it was not possible to exactly determine the axonal or extraaxonal location of these depositions by light 


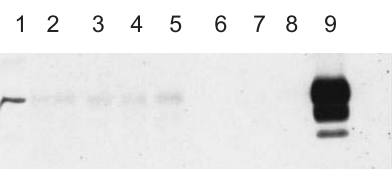

A. $42 \mathrm{dpi}$

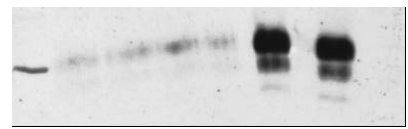

B. $60 \mathrm{dpi}$
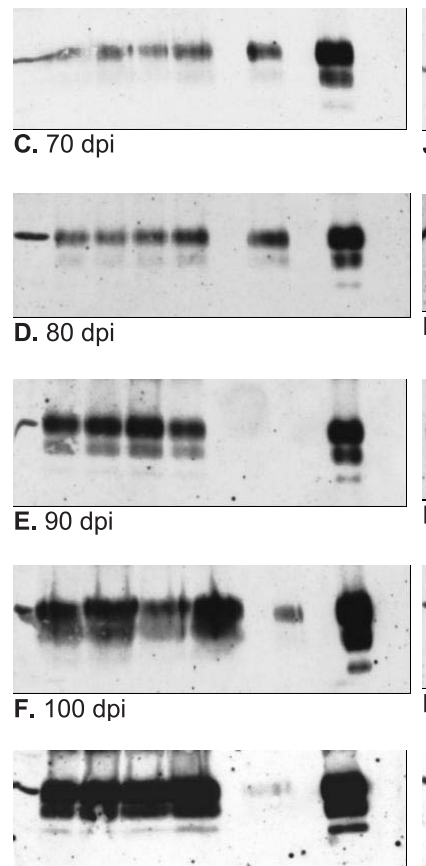

G. 126 dpi (terminally ill)

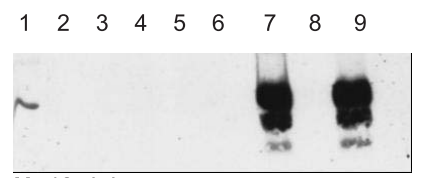

H. $42 \mathrm{dpi}$

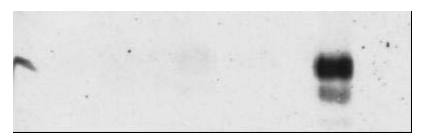

I. $60 \mathrm{dpi}$
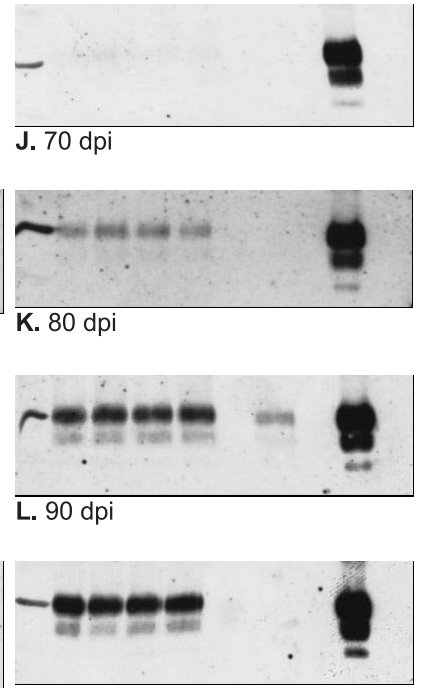

M. 100 dpi

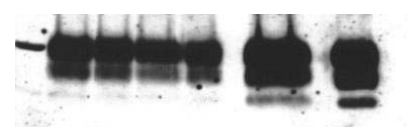

N. 126 dpi

Figure 6. Detection of PrP27-30. Representative Western blots of ipsilateral (A-G) and contralateral $(\mathrm{H}-\mathrm{N})$ sciatic nerve tissue of $263 \mathrm{~K}$ f.p. infected and IDPN-treated hamsters for indicated time points after infection. Lane 1: marker; lanes 2-5: sciatic nerve quarters from proximal to distal of the same individual at the indicated time point (samples correspond to the following amounts of tissue $(\mathrm{mg})$ : $\mathrm{A} 2$, 2.6; A3, 2.5; A4, 2.9; A5, 4.6; B2, 1.2; B3, 1.0; B4, 1.5; B5, 1.9; C2, 2.5; C3, 2.1; C4, 2.0; C5, 3.7; D2, 2.9; D3, 1.9; D4, 2.9; D5, 3.3; E2, 6.2; E3, 4.3; E4, 4.5; E5, 5.5; F2, 3.9; F3, 3.3; F4, 3.5; F5, 5.6; G2, 2.6; G3, 2.1; G4, 1.9; G5, 2.8; H2, 5.4; H3, 2.8; H4, 4.4; H5, 4.0; I2; 1.8; I3, 1.7; I4, 1.4; I5, 1.6; J2, 2.1; J3, 2.2; J4, 2.2; J5, 4.6; K2, 3.4; K3, 3.3; K4, 3.4; K5, 4.4; L2, 4.9; L3, 4.1; L4, 3.4; L5, 5.0; M2, 4.7; M3, 3.1; M4, 3.3; M5, 4.0; N2, 3.1; N3, 1.7; N4, 2.6; N5, 4.8); lane 7 (B, C, $\mathrm{D}, \mathrm{F}, \mathrm{H}, \mathrm{L}, \mathrm{N}$ ): spiked processing controls, $10^{-5} \mathrm{~g} 263 \mathrm{~K}$ brain homogenate (samples correspond to the following amounts of normal sciatic nerve tissue (mg): B7, 2.5; C7, 2.7; D7, 3.5; F7, 2.5; H7, 2.4; L7, 1.9; N7, 2.7); lane 7 (A, E, G, I, J, K, M): uninfected nerves as controls for PK digestion (samples correspond to the following amounts of normal sciatic nerve tissue (mg): A7; 3.6; E7, 1.6; $\mathrm{G} 7,2.2 ; \mathrm{I} 7,3.0 ; \mathrm{J} 7,2.3 ; \mathrm{K} 7,2.7$; M7, 2.1); lane 9: blot controls, $10^{-6} \mathrm{~g} 263 \mathrm{~K}$ brain homogenate. 
microscope. Groschup et al. [24] suggested adaxonal location of $\mathrm{PrP}^{\mathrm{Sc}}$ deposits in peripheral nerves for the i.p. route of infection.

Western blot signals for PrP27-30 from the sciatic nerve segments did not indicate any clear direction of $\mathrm{PrP}^{\mathrm{Sc}}$-propagation: the blot signal intensities from all segments of a unilateral nerve in the same individual at one time point were almost equal (Figs. 1 and 6). This may be due to the limited resolution of the detection method and/or to the comparative observations along a relatively short neural distance (quarters of a length of 2-3 cm nerve). Another possible explanation may be that the infectious agent rapidly passes neural tissue at earlier points in time of the incubation period, something that is also assumed for vagal transit [35].

Another objective of this study was to identify or narrow down potential mechanisms for the neural centripetal/centrifugal propagation of the TSE agent. In order to determine whether this propagation is dependent on an intact axonal cytoskeleton we studied the spread of scrapie in IDPN intoxicated animals. The effects of IDPN as a toxin causing changes in cytoskeletal architecture and in axonal transport of proteins are well characterized by various in vivo models including a broad range of species (e.g. rats, cats, chickens, guinea pigs). IDPN treatment induces structural abnormalities in axonal segments $[14,15]$, neurofilaments displace toward the axonal periphery while microtubuli displace toward the axonal center [23, 37]. Giant axonal swellings appear, containing maloriented neurofilaments [16]. In motor end plates, the immunoreactivity of neurofilaments is decreased [18]. The neuronal cytoskeleton in IDPN-treated animals can be characterized as "disorganized". Several data indicate that axonal transport mechanisms are impaired: predominantly the slow anterograde ones but also the retrograde and fast ones $[19,21,30,38]$. Com- pared to the doses described in the literature for experiments with various species, the administered dose in the present study ranged in the lower level. Chronic low dose intoxication with IDPN in our test animals induced an increased immunoreactivity of neurofilaments in sections of the distal sciatic nerve, confirming an effect on the axonal cytoskeleton. Regardless of possible changes in the phosphorylation status of the neurofilament proteins leading to a possibly higher affinity to the antibody labelling of the neurofilaments, we would interpret this observation as indicating an enrichment of neurofilaments in distal axons, analogous to results in IDPN-intoxicated cats [22]. Moreover, based on visual subjective judgement, the axonal diameter seemed to be slightly increased (Fig. 4). The occurrence of salivation shortly after i.p. application in hamsters could be the effect of the neurotoxin on neurotransmitters involved in the control of salivary gland secretion. The quality and quantity of the animals' white blood cells suggested that there was no evidence of general inflammation caused by IDPN-treatment regime. Inflammations were shown to have an influence on the detection of $\mathrm{PrP}^{\mathrm{Sc}}$ or on scrapie pathogenesis [26].

A comparison of the incubation times in neurotoxin exposed and unexposed animals (Fig. 5) led to the assumption that the neuroinvasive progression of disease is independent of the neurofilament component of the axonal cytoskeleton. Moreover, the comparison of the results of spatiotemporal $\mathrm{PrP}^{\mathrm{Sc}}$ detection in sciatic nerves without or with IDPN treatment (Figs. 1 and 6) did not indicate any influence of the axonal neurofilament component on the propagation of the pathological prion protein in peripheral nerves.

One conceivable mode of transportation along peripheral nerves for the abnormal prion protein is the host's axonal transport machinery. Although the molecular basis is not completely understood, members 
of the Herpesviridae family are examples of agents using this transport system [50] as well as neurotoxins (i.e. tetanus toxin, [41]). IDPN intoxication mainly affects the slow axonal transport mechanism. Regarding the relevance of neurofilaments for slow component ' $a$ ' [9], the IDPN model has the potential to specify the influence of host slow axonal transport mechanism 'a' on propagation of $\mathrm{PrP}^{\mathrm{Sc}}$. Although the degree of impairment of the axonal flow achieved by IDPN-intoxication was not monitored in this study, it is strongly indicated that axonal propagation of $\mathrm{PrP}^{\mathrm{Sc}}$ does not correlate with the slow axonal transport system component ' $a$ '.

Finally, we only focused on the neuronal pathway and its structural-functional modification in one TSE in vivo model. Alternative pathways of spreading should be examined, too, including simultaneous observations of other tissues. Further investigations are necessary to elucidate the impact of neuronal vs. nonneuronal pathways for the footpad model.

\section{ACKNOWLEDGEMENTS}

This work was supported by the European Union (Contract No. QLG3-CT-2002-81030). We thank Kristin Kampf, Marion Joncic and Manuela Friedrich for their excellent technical assistance. Sincere thanks are also given to Walter Schulz-Schaeffer and Tatjana Pfander for the introduction to histological methods.

\section{REFERENCES}

[1] Alpers M.P., Epidemiology and ecology of kuru, in: Prusiner S.B., Hadlow W.J. (Eds.), Slow transmissible diseases of the nervous system, Academic Press, New York, Vol. 1, 1979, pp. 67-92.

[2] Anderson R.M., Donnelly C.A., Ferguson N.M., Woolhouse M.E., Watt C.J., Udy H.J., MaWhinney S., Dunstan S.P., Southwood T.R., Wilesmith J.W., Ryan J.B., Hoinville L.J., Hillerton J.E., Austin A.R., Wells G.A.,
Transmission dynamics and epidemiology of BSE in British cattle, Nature (1996) 382:779-788.

[3] Andreoletti O., Berthon P., Marc D., Sarradin P., Grosclaude J., van Keulen L., Schelcher F., Elsen J.M., Lantier F., Early accumulation of $\operatorname{PrP}(\mathrm{Sc})$ in gut-associated lymphoid and nervous tissues of susceptible sheep from a Romanov flock with natural scrapie, J. Gen. Virol. (2000) 81:3115-3126.

[4] Bartz J.C., Kincaid A.E., Bessen R.A., Retrograde transport of transmissible mink encephalopathy within descending motor tracts, J. Virol. (2002) 76:5759-5768.

[5] Bartz J.C., Kincaid A.E., Bessen R.A., Rapid prion neuroinvasion following tongue infection, J. Virol. (2003) 77:583-591

[6] Beekes M., Baldauf E., Cassens S., Diringer H., Keyes P., Scott A.C., Wells G.A., Brown P., Gibbs C.J. Jr., Gajdusek D.C., Western blot mapping of disease-specific amyloid in various animal species and humans with transmissible spongiform encephalopathies using a high-yield purification method, J. Gen. Virol. (1995) 76:2567-2576.

[7] Beekes M., McBride P.A., Baldauf E., Cerebral targeting indicates vagal spread of infection in hamsters fed with scrapie, J. Gen. Virol. (1998) 79:601-607.

[8] Borchelt D.R., Koliatsos V.E., Guarnieri M., Pardo C.A., Sisodia S.S., Price D.L., Rapid anterograde axonal transport of the cellular prion glycoprotein in the peripheral and central nervous systems, J. Biol. Chem. (1994) 269:14711-14714.

[9] Brown A., Axonal transport of membranous and nonmembranous cargoes: a unified perspective, J. Cell Biol. (2003) 160:817-821.

[10] Brown P., Preece M., Brandel J.P., Sato T., McShane L., Zerr I., Fletcher A., Will R.G., Pocchiari M., Cashman N.R., d'Aignaux J.H., Cervenakova L., Fradkin J., Schonberger L.B., Collins S.J., Iatrogenic Creutzfeldt-Jakob disease at the millennium, Neurology (2000) 55:1075-1081.

[11] Bueler H., Aguzzi A., Sailer A., Greiner R.A., Autenried P., Aguet M., Weissmann C., Mice devoid of PrP are resistant to scrapie, Cell (1993) 73:1339-1347.

[12] Carp R.I., Transmission of scrapie agent by oral route: effect of gingival scarification, Lancet (1982) 16:170-171.

[13] Castilla J., Saa P., Hetz C., Soto C., In vitro generation of infectious scrapie prions, Cell (2005) 121:195-206. 
[14] Chou S.M., Hartmann H.A., Axonal lesions and waltzing syndrome after IDPN administration in rats. With a concept"AXOSTASIS", Acta Neuropathol. (Berl.) (1964) 40:428-450.

[15] Chou S.M., Hartmann H.A., Electron microscopy of focal neuroaxonal lesions produced by beta-beta-iminodipropionitrile (IDPN) in rats. I. The advanced lesions, Acta Neuropathol. (Berl.) (1965) 4:590-603.

[16] Clark A.W., Griffin J.W., Price D.L., The axonal pathology in chronic IDPN intoxication, J. Neuropathol. Exp. Neurol. (1980) 39:42-55.

[17] Eklund C.M., Kennedy R.C., Hadlow W.J., Pathogenesis of scrapie virus infection of the mouse, J. Infect. Dis. (1967) 117:15-22.

[18] Ferri G.L., Cichi A., Bastone A., Gaudio R.M., Frontali N., Dahl D., Experimental beta beta'-iminodipropionitrile (IDPN) neuropathy: neurofilament profile of sensory, motor and autonomic nerves as seen by immunocytochemistry on whole-mount preparations, Brain Res. (1994) 657:315-319.

[19] Fink D.J., Purkiss D., Mata M., beta,beta' Iminodipropionitrile impairs retrograde axonal transport, J. Neurochem. (1986) 47:1032-1038.

[20] Gajdusek D.C., Hypothesis: interference with axonal transport of neurofilament as a common pathogenetic mechanism in certain diseases of the central nervous system, N. Engl. J. Med. (1985) 312:714-719.

[21] Griffin J.W., Hoffman P.N., Clark A.W., Carroll P.T., Price D.L., Slow axonal transport of neurofilament proteins: impairment of beta,beta'-iminodipropionitrile administration, Science (1978) 202:633-635.

[22] Griffin J.W., Gold B.G., Cork L.C., Price D.L., Lowndes H.E., IDPN neuropathy in the cat: coexistence of proximal and distal axonal swellings, Neuropathol. Appl. Neurobiol. (1982) 8:351-364.

[23] Griffin J.W., Fahnestock K.E., Price D.L., Hoffman P.N., Microtubule-neurofilament segregation produced by beta, beta'iminodipropionitrile: evidence for the association of fast axonal transport with microtubules, J. Neurosci. (1983) 3:557-566.

[24] Groschup M.H., Beekes M., McBride P.A., Hardt M., Hainfellner J.A., Budka H., Deposition of disease-associated prion protein involves the peripheral nervous system in experimental scrapie, Acta Neuropathol. (1999) 98:453-457.
[25] Hafezparast M., Brandner S., Linehan J., Martin J.E., Collinge J., Fisher E.M., Prion disease incubation time is not affected in mice heterozygous for a dynein mutation, Biochem. Biophys. Res. Commun. (2005) 326:18-22.

[26] Heikenwalder M., Zeller N., Seeger H., Prinz M., Klohn P.C., Schwarz P., Ruddle N.H., Weissmann C., Aguzzi A., Chronic lymphocytic inflammation specifies the organ tropism of prions, Science (2005) 307:11071110 .

[27] Kascsak R.J., Rubenstein R., Merz P.A., Tonna-DeMasi M., Fersko R., Carp R.I., Wisniewski H.M., Diringer H., Mouse polyclonal and monoclonal antibody to scrapieassociated fibril proteins, J. Virol. (1987) 61:3688-3693.

[28] Kimberlin R.H., Walker C.A., Pathogenesis of mouse scrapie: evidence for neural spread of infection to the CNS, J. Gen. Virol. (1980) 51:183-187.

[29] Kimberlin R.H., Hall S.M., Walker C.A., Pathogenesis of mouse scrapie. Evidence for direct neural spread of infection to the CNS after injection of sciatic nerve, J. Neurol. Sci. (1983) 61:315-325

[30] Komiya Y., Cooper N.A., Kidman A.D., The long-term effects of a single injection of beta,beta'-iminodipropionitrile on slow axonal transport in the rat, J. Biochem. (Tokyo) (1986) 100:1241-1246.

[31] Kunzi V., Glatzel M., Nakano M.Y., Greber U.F., Van Leuven F., Aguzzi A., Unhampered prion neuroinvasion despite impaired fast axonal transport in transgenic mice overexpressing four-repeat tau, J. Neurosci. (2002) 22:7471-7477.

[32] Legname G., Baskakov I.V., Nguyen H.O., Riesner D., Cohen F.E., DeArmond S.J., Prusiner S.B., Synthetic mammalian prions, Science (2004) 305:673-676

[33] Llewelyn C.A., Hewitt P.E., Knight R.S., Amar K., Cousens S., Mackenzie J., Will R.G., Possible transmission of variant Creutzfeldt-Jacob disease by blood transfusion, Lancet (2004) 363:417-421.

[34] McBride P.A., Beekes M., Pathological PrP is abundant in sympathetic and sensory ganglia of hamsters fed with scrapie, Neurosci. Lett. (1999) 265:135-138.

[35] McBride P.A., Schulz-Schaeffer W.J., Donaldson M., Bruce M., Diringer H., Kretzschmar H.A., Beekes M., Early spread of scrapie from the gastrointestinal tract to 
the central nervous system involves autonomic fibers of the splanchnic and vagus nerves, J. Virol. (2001) 75:9320-9327.

[36] Moya K.L., Hassig R., Creminon C., Laffont I., Di Giamberardino L., Enhanced detection and retrograde axonal transport of $\mathrm{PrPc}$ in peripheral nerve, J. Neurochem. (2004) 88:155-160.

[37] Papasozomenos S.C., Autilio-Gambetti L., Gambetti P., Reorganization of axoplasmic organelles following beta, beta'-iminodipropionitrile administration, J. Cell Biol. (1981) 91:866-871.

[38] Papasozomenos S.C., Yoon M., Crane R., Autilio-Gambetti L., Gambetti P., Redistribution of proteins of fast axonal transport following administration of beta,beta'-iminodipropionitrile: a quantitative autoradiographic study, J. Cell Biol. (1982) 95:672-675.

[39] Pattison I.H., Millson G., Experimental transmission of scrapie to goats and sheep by the oral route, J. Comp. Pathol. (1961) 71:171-176.

[40] Peden A.H., Preclinical vCJD after blood transfusion in a PRNP codon 129 heterozygous patient, Lancet (2004) 364:527-529.

[41] Price D.L., Griffin J., Young A., Peck K., Stocks A., Tetanus toxin: direct evidence for retrograde intraaxonal transport, Science (1975) 188:945-947.

[42] Prusiner S.B., Novel proteinaceous infectious particles cause scrapie, Science (1982) 216:136-144.
[43] Prusiner S.B., Cochran S.P., Alpers M.P., Transmission of scrapie in hamsters, J. Infect. Dis. (1985) 152:971-978.

[44] Radebold K., Chernyak M., Martin D., Manuelidis L., Blood borne transit of CJD from brain to gut at early stages of infection, BMC Infect. Dis. (2001) 1:20.

[45] Rodolfo K., Hassig R., Moya K.L., Frobert Y., Grassi J., Di Giamberardino L., A novel cellular prion protein isoform present in rapid anterograde axonal transport, Neuroreport (1999) 10:3639-3644.

[46] Sigurdson C.J., Williams E.S., Miller M.W., Spraker T.R., O'Rourke K.I., Hoover E.A., Oral transmission and early lymphoid tropism of chronic wasting disease PrPres in mule deer fawns (Odocoileus hemionus), J. Gen. Virol. (1999) 80:2757-2764.

[47] Stahl N., Borchelt D.R., Hsiao K., Prusiner S.B., Scrapie prion protein contains a phosphatidylinositol glycolipid, Cell (1987) 51:9229-9240.

[48] Taylor D.M., McConnell I., Fraser H., Scrapie infection can be established readily through skin scarification in immunocompetent but not immunodeficient mice, J. Gen. Virol. (1996) 77:1595-1599

[49] Thomzig A., Kratzel C., Lenz G., Krüger D., Beekes M., Widespread PrPSc accumulation in muscles of hamsters orally infected with scrapie, EMBO Rep. (2003) 4:530-533.

[50] Tomishima M.J., Smith G.A., Enquist L.W., Sorting and transport of alpha herpesviruses in axons, Traffic (2001) 2:429-436. 